\title{
Software Design Collection and Handling of Signal Sound Body
}

\author{
Son Nguyen Van, Duc Trinh Quang, Giang Nguyen Hoai, Quynh Nguyen Thi Huong, Khanh Pham Xuan
}

\begin{abstract}
We demonstrate the designed software that possibly collects the body sound data to be used for clinical diagnosis applications. Body sound signals are collected and processed through a software designed in Labview to adapt with Arduino-Uno. The analog signals transduced from a piezoelectric microphone are converted to the digital signals by an ADC component integrated in the Uno board and controlled the sampling frequency via the software. The collected signals are observed and visualized in graph panel of the software and the audio sound can play through speakers in real-time then stored the measured values as the audio file format simultaneously. The data can use to analyze by another software or study the analyzed algorithm to extract the disease signals. To evaluate the quality of the system, a series of experiments were examined in hospital environment and asserted with clinical experiences of specified medical doctors. To enhance the scope of the disease signal, the spectrum of the signal can be collected ranged on $5 \mathrm{~Hz}$ to $35 \mathrm{kHz}$ corresponding to the full spectrum of the hardware system, with the sampling frequency reached to $100 \mathrm{kHz}$. Based on this initial system, a series of development applying to clinical diagnosis can be potentially opened in the near future.
\end{abstract}

Keywords: Digital Stethoscope, Body sound, Labview, signal processing.

\section{INTRODUCTION}

Body sound is one of the important clinical signs for disease diagnosis, discovered in the early 18th century [1]. Because sound can be transmitted directly out of the body through biological epitheles with little obstruction, this clinical manifestation can be heard directly by the doctor by ear. The body's acoustic signal is very small, can only be heard loudly about 30 to $40 \mathrm{~dB}$ [2]. In order to amplify these sounds, acoustic amplifiers were made with amplification of about 2 $\mathrm{dB}$ [2]. Traditional stethoscope is designed to be sensitive enough for doctors to use, however, these data are difficult to store and share.

Revised Manuscript Received on December 30, 2019.

* Correspondence Author

Son Nguyen Van*, Hanoi Open University, Hanoi, Vietnam..

Duc Trinh Quang, Hanoi University of Science and Technology, Hanoi, Vietnam.

Giang Nguyen Hoai, Hanoi Open University, Hanoi, Vietnam.

Quynh Nguyen Thi Hương, Department of Science, Technology and EnvironmentDepartment, Ministry of Education and Training

Khanh Pham Xuan, Hanoi Vocationa College of High Technoogy

(C) The Authors. Published by Blue Eyes Intelligence Engineering and Sciences Publication (BEIESP). This is an open access article under the CC BY-NC-ND license (http://creativecommons.org/licenses/by-nc-nd/4.0/)
With the speed of development of telecommunication system today, remote diagnosis as well as storage of clinical information of patients for long-term follow-up in electronic medical records is one of the requirement. In consider of the demand to digitize the body's audio signals for storage and use in the data for remote disease diagnosis, electronic medical monitoring, and visualization in teaching and medical training, the electronic stethoscope had been built [3]. The market for medical devices initially approved the stethoscope with even it was expensive and complicated to use because of its benefits, especially for the demanding of remote medical consultation and treatment.

Recently, electronic stethoscope becomes popularized in hospital equipment and clinics [4] [5]. Typically, the electronic stethoscope includes analog bandpass filter, amplifier, digital bandpass filter and zero-adjustable circuit. The acoustic-electrical signal is firstly filtered by the analog filter then amplified, zero adjusted, digitized, and refined by digital filtered. After the signal is processed, the audio is played through speaker or store in a storage device. For the storage purpose and analysis using software computer, the interface to the computer is required, hence, the device should be designed with a adapted PC connections such as USB, Bluetooth or via a wireless local network called Wifi. In the framework of this report, a software designed to collect signals from a measured device to computer through USB port will be presented. Software is written in Labview environment.

\section{MATERIALS AND METHODS}

\subsection{Materials}

The electronic stethoscope used in this report is built with a wide-spectrum probe, from $5 \mathrm{~Hz}$ to $40 \mathrm{kHz}$. The sensor is placed in a resonant chamber that mimics the acoustic amplification drum of a traditional electronic stethoscope. The acoustic-electric signal is amplified by an amplifier with a gain of $40 \mathrm{~dB}$ to $100 \mathrm{~dB}$ and a signal-to-noise ratio (SNR) reaches to $50 \mathrm{~dB}$. Since the quality of the test signal does not affect the outcome of the software design, this report does not describe in detail the design of an analog amplifier circuit. The digitization of the signal is also through the sampling of a modularized microcontroller into a commercial product manufactured by Arduino. The digitized signal is sampled by the computer's sampling frequency when transmitting parameters to the Arduino module. Digitized data will be transmitted from Arduino to the computer via USB interface. 
The main programming tool used here is the graphical programming language, in which functions and procedures are modularized into blocks. The programmer connects these blocks together according to the algorithm designed to form the software.

\subsection{Method}

The software is designed to include: Signal conversion interface, digital filtering, signal display, live listening and storage. The function blocks are arranged as shown in Figure 1. The signal has been digitized by the microcontroller built into the Arduino module which is communicated with the computer via the signal conversion interface module. This module is responsible for ordering the Arduino microcontroller to send data and receive sampling frequency parameters via the USB communication port. After the digital signal is sent to the computer, these data are stored as a stack the computer's memory.

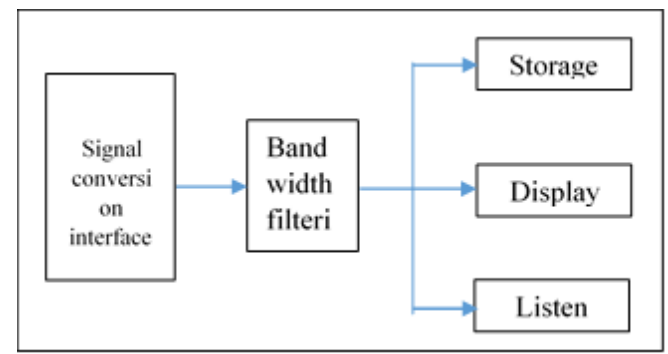

Fig. 1. Diagram of the software block system.

From this data array, the data collected is plotted by graph tool on a computer and can be heard via the audio port of the computer via the listening module. At the end of the sampling process that displays, numeric array data can be stored through the storage module. In order to expand the application, the storage module does not encrypt the signal but stores it as a decimal array which is the measurement value in real time voltage and unit. These data can be read and processed by specialized software for data analysis such as Matlab, Excel, Mathematica ...

\section{THE SOFTWARE MODULE}

The graphical programming tool used in this software design is Labview, a very powerful programming tool for creating desktop applications entirely in graphical language. Procedures and sub functions are packaged into modules whose input and output are specified in predefined data format. The programmer simply takes these blocks out and joins them in an algorithmic sequence. Initially, this tool was only developed for National Instrument hardware devices; however, other sub procedures were developed by the Labview user community. Labview-based applications are often very convenient in developing measurement and control tools.

\subsection{Digital conversion interface module}

The digital conversion interface module consists of 4 connected procedures as shown in Figure 2: the communication terminal, the configuration unit for the device (here is Arduino), the data acquisition unit and the procedure termination block. . These blocks are developed based on subprograms that have been completed by some authors for common hardware modules for Labview. Based on the VISA library, support for Arduino's Uno module has been developed to make communication easier.

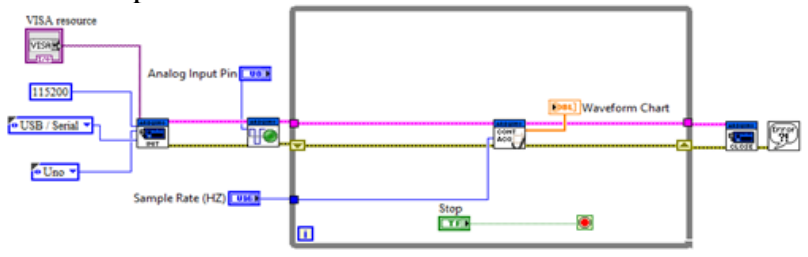

Fig. 2. Program of digital conversion interface module.

In the initialization procedure, the USB device is used, the data transmission form is the serial frame, the transmission speed is set to 115200 bits / sec. From this initialization block, the computer is confirmed to have shook hands with the Uno module. Through the configuration block, the Uno analog input channel is marked so that the Uno module control can digitize the signal. In this system, Uno channel 0 is used among 8 analog input channels. The digitalization unit is controlled by the sampling frequency, in this system, the sampling frequency is set to $100 \mathrm{kHz}$ to fit the sound range from 0 to $40 \mathrm{kHz}$ according to the Nyquist standard. The output of this block is the data that is stored in a compact form for continuous display by Labview's graph tool. The end of the procedure is performed when the data collection loop ends to ensure that the software can interrupt data collection.

\subsection{Band pass filter}

The body's audio signals that the system cares about are sounds within the audible range. For research in medical acoustics, the preferred spectrum is in the range of 0 to $8 \mathrm{kHz}$. These sounds include the respiratory, cardiovascular, and digestive systems. To date, respiratory and cardiovascular sounds have been extensively studied, but gastrointestinal sound is still an open field due to complexity in biological mechanisms.

With the preferred frequency segment, the elimination of signals in the DC (DC) and high frequency (above $40 \mathrm{kHz}$ ) regions is necessary to increase the signal to noise ratio (SNR) as well as create contrast when observing the spectrum. of the signal. To accomplish this function, the band gap filter is designed with Labview source code as shown in Figure 3.

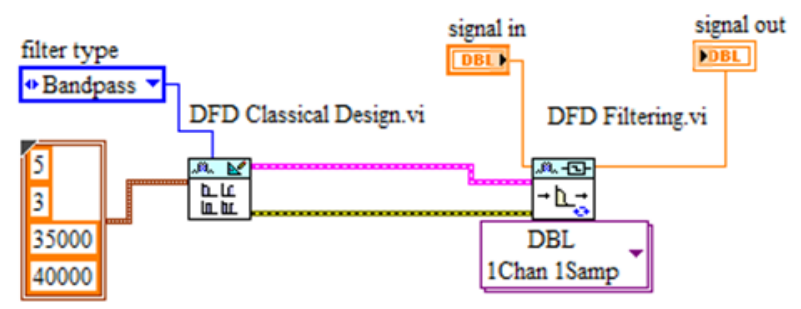

Fig. 3. Labview source code of the bandpass filter module

For analogs, implementing a filter with a high cutoff factor is not straightforward because it requires a higher order of the filter while the nonlinearity of the component is quite high. Parameter filter allows to perform complex calculations easily, the signal is the value multiplied by the cutoff frequency. The cutoff characteristic of the high pass filter part here has a cutoff frequency of $3 \mathrm{~Hz}$ and a stop frequency of $5 \mathrm{~Hz}$, while the cutoff frequency of the low pass filter section is $35 \mathrm{kHz}$ and the stop frequency of the filter is $40 \mathrm{kHz}$. 
The order of the filter here does not need to be considered because since the cut and stop frequency, the filter procedure will automatically calculate the sample and element coefficients of the filter so that the filter order will determine. The configuration of the filter used here is Butterworth to ensure no margin of error.

\subsection{Display Module}

Labview has built in handy display modules in many forms, 1D to display 1-dimensional arrays, 2D to display 2-dimensional arrays and 3D to display surfaces or blocks. For the purpose of observing the audio signal collected from the microcontroller, 1D display is used. The window in Figure 4 is a display of numerical data values.

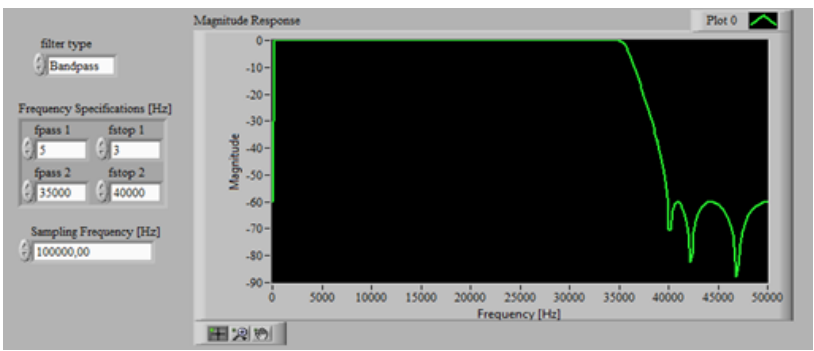

Fig. 4. Graphical representation of the audio data array

Digital data is connected together continuously. While the digital conversion interface module continuously takes new data, the previously collected data is still stored in FILO (first in and out) format in memory and arranged in arrays. These figures are plotted continuously on the display screen to facilitate the viewer to observe the form of the signal. On the left corner are the parameters for configuring the bandpass filter module.

\subsection{Live Listening Audio Block}

Because data is sound, so visually to the observer is sound. To create this visualization, the software used DAC (digital analog converter) of computers integrated on the soundcard. Through the existing procedures developed on Labview, digital data is pushed through the DAC of the soundcard through the procedures: signal configuration, amplification of volume adjustment, data array creation and DAC output.

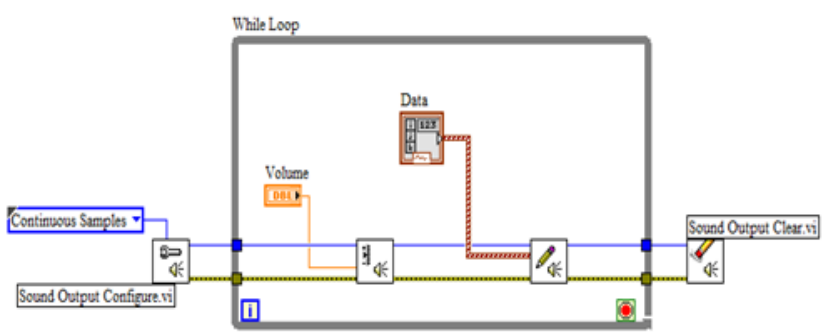

Fig. 5. Labview source code of the live audio listening module

The source code of the live listening module is shown in Figure 5, consisting of 4 blocks as stated above. Data is defined in the form of continuous sound. Amplification is changed via volume shown in the form of a knob. The data is passed to the array generator and this block will push this sub array at the DAC output. Because common peripherals of computers are installed with Windows operating system drivers, Labview will use these functions to control peripheral devices. In the absence of such drivers, control subroutines can be performed via Labview's VISA toolbox.

\subsection{Storage module}

The source code of the storage module is shown in Figure 6. The data stored in memory after the measurement is completed will be passed through the sound file write procedure to create the file. The data includes 2 fields: measurement value and real time taken on the computer. End of file recording is the procedure of packaging and ending files. The data format here is not encrypted and is simply a text file that can be used by other analytics software.

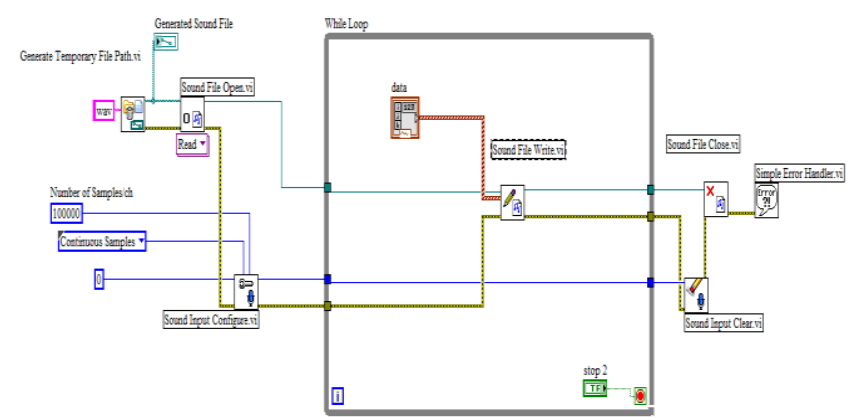

Fig. 6. Source code of the storage module

This is also one of the important tools since the commercial software of digital stethoscope cannot afford due to the confidentiality of manufacturers. Moreover, the recorded data are encrypted so that other common software cannot be used. In order to extract data, users need to purchase software tools to develop their own analytical algorithms.

\section{RESULT}

The software and hardware of the digital listening device were tested at Hanoi Heart Hospital and collected results from volunteers and patients. The measurement is performed by the following steps:

1- Listen to the signal directly and observe the waveform of the signal on the display.

2- When determining the characteristic of repetitive signal at least 2 times, stop measuring.

3- Record data with clinical manifestations recorded in the file name.

Figure 7 shows data from a volunteer's healthy state. Data are measured at intervals of 15 seconds.

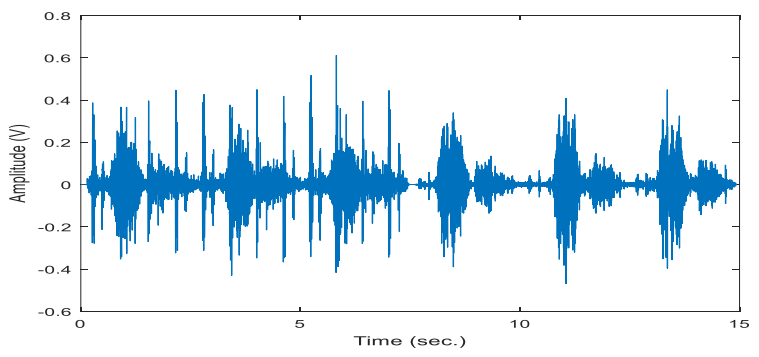

Fig. 7. Lung sounds of a healthy volunteer 
The waves are characterized by sound waves that have a fairly regular beat, in cycles of about 2.5 seconds. In each cycle, there are 2 characteristic buds with a high amplitude and a low amplitude. In between 3 continuous breaths is a signal space of about 0.5 seconds. The characteristics of the signals are the amplitude of the repetition of the uniform waves and the value without much change.Figure 8 shows a signal of a patient with mild bronchitis. The breathing is also steady and lasts for about 2.5 seconds to 3 seconds. Unlike the sound of a healthy volunteer, the characteristic signal of the signal is the high and low wave buds symbolizing the elimination and exhalation cycles of the lung. The average amplitude of the signal is also unstable. This feature can be explained by the slightly inflamed air passage through the trachea due to the swelling of the inner lining of the bronchus and narrowing of the bronchial area at the site of inflammation.

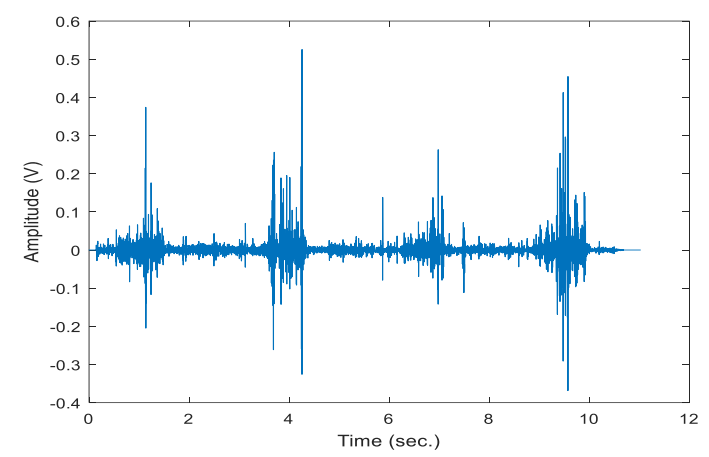

Fig. 8. Data of patients with mild bronchitis.

Figure 9 is a data of patients with chronic bronchitis. In the form of data collected also includes 4 breathing cycles. However, different from the data of patients with mild bronchitis as well as healthy volunteers, in patients with chronic bronchitis the boundary between breathing patterns is blurred. Clinical data also shows that the patient's mucosa is sclerotic after many inflammatory episodes, so the continuous flow of air through the narrowing of the bronchial portion of the bronchi after the end of each audible breathing rhythm and quite large amplitude. This is the reason why there are still sounds coming from the bronchus between the breaths. For patients who have not yet been mucosalized, the elasticity of the mucosa also reduces the change in airflow rate resulting in a slight change in the flow of sound waves.
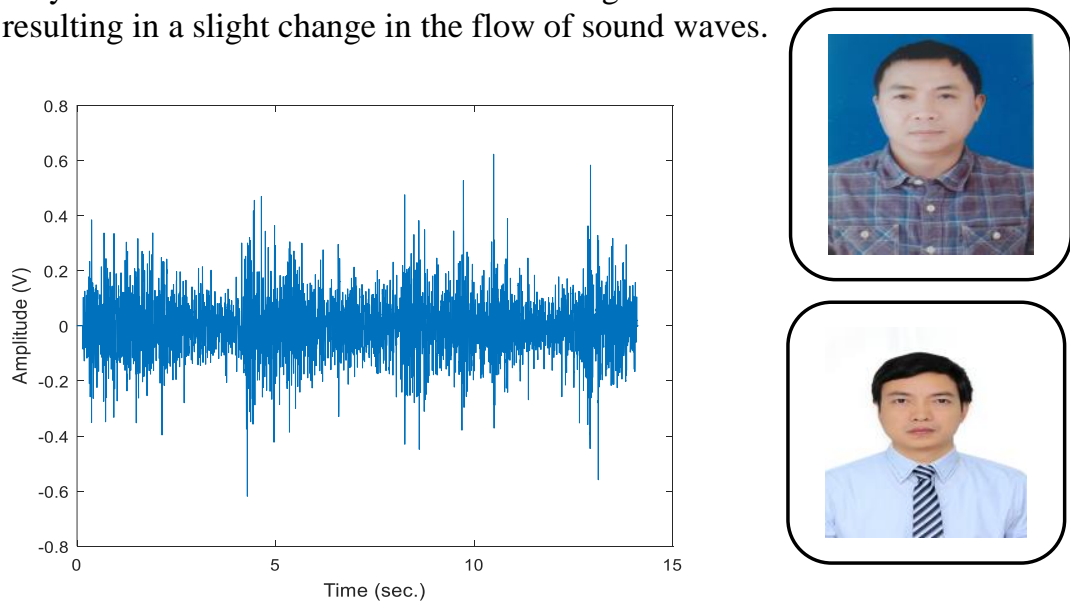

interpreted by specialists to show that the system of sound recording systems ensures the qualitative and quantitative of signal.

\section{CONCLUSION}

The body's audio signals are collected, processed, displayed and visualized such as listening and graph observation directly, then, allowing to be stored in computers in the form of open data encapsulated in a Design software based on Labview graphics programming language. The software can interact directly with the Arduino-Uno hardware module to control the process of collecting signals, handling DC noise and high-frequency interference with a band-pass filter. The visual display through graphs and real-time audio makes the observer convenient. With 5 to 35 $\mathrm{kHz}$ bandwidth filter and $100 \mathrm{kHz}$ sampling frequency, the device can fully capture audio from high frequency to high frequency range. To illustrate the system, the data from volunteers and patients is also confirmed by clinical trials using hospital settings.

\section{ACKNOWLEDGMENT}

This work is financially supported by the program named as B2017-MHN.01 of Vietnam Ministry of Education and Trainning.

\section{REFERENCES}

1. Laennec, R. T. H.; Forbes, John, Sir, A Treatise on the Diseases of the Chest and on Mediate Auscultation. New York : Samuel Wood \& Sons ; Philadelphia : Desilver, Thomas \& Co. 1835Lieberman, Karen MS, CRNP, "Interpreting 12-Lead ECGs: A Piece by Piece Analysis", October 2008, Volume 33 Number 10 , p 28 - 35.

2. Wade, Nicholas J.; Deutsch, Diana. Binaural Hearing - Before and After the Stethophone. Acoustics Today: 16-27. 2008.

3. The story behind the development of $3 \mathrm{M}$ Littmann Electronic Stethoscopes, 3M, Issued 8/11, 8102HB 70-2010-8403-8, 2011.

4. GosReports. Global Stethoscopes Market Research Report 2016, December 30, 2016.

5. Global Industry Analysts, Inc. Advanced Electronic Innovations to Benefit Global Sales of the Iconic Stethoscopes Which Continue to Remain Indispensable to Medical Practice. MCP-3363, April 2017.

\section{AUTHORS PROFILE}

Son Nguyen Van is a lecturer of Faculity of Electronic Technology and Communication, Hanoi Open University. He received his B.E, M.S. degrees in Eelectronics and Telecommunications at Hanoi Open University, Vietnam

Duc Trinh Quang received his B.E, M.S. degrees in Control Engineering and Automation at HUST, Vietnam, and Ph.D. degrees (2012) in Tohoku Institute of Technolog, Sendai, Japan, he is lecturer of Electronics and Telecommunications at HUST, Vietnam

Fig. 9. Data of patients with chronic bronchitis

The three basic data collected together with the clinical data collected and the specificity of the sound were 


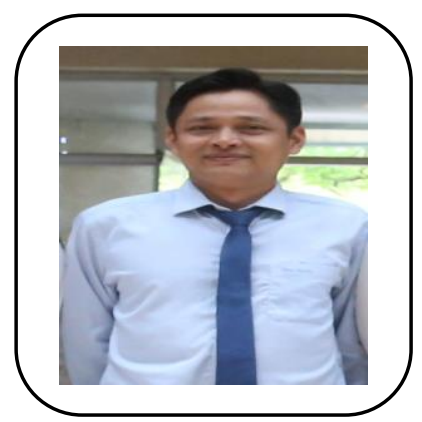

Giang Nguyen Hoai received his B.E, M.S. degrees in Information Technology at Budapest University of Technology and Economics, Hungary, and Ph.D. degrees (2009) in Electronics and Telecommunication from Hanoi University of Science and Technology (HUST). He is lecturer of Eelectronics and Telecommunications at Hanoi Open University, Vietnam

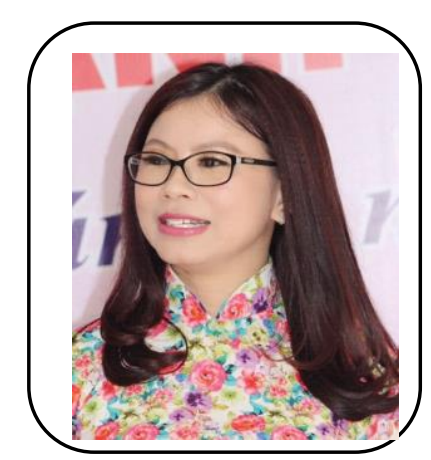

Quynh Nguyen Thi Huong works at the Department of Science, Technology and Environment, Vietnam Ministry of Education and Training. She has a degree in Information Technology and is studying for a $\mathrm{PhD}$ in science management

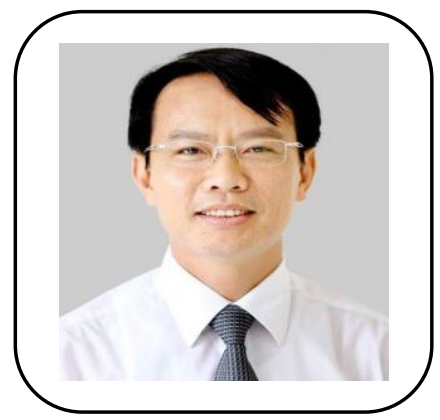

Khanh Pham Xuan, received his B.E degrees in electronics, M.S. degrees in information technology, and Ph.D degrees (2012) at HUST, Vietnam. He is lecturer of

Hanoi Vocationa College of High Technoogy, Vietnam 